\title{
ON SELF-INJECTIVE ALGEBRAS OF STABLE DIMENSION ZERO
}

\author{
MICHIO YOSHIWAKI
}

\begin{abstract}
Let $A$ be a self-injective algebra over an algebraically closed field. We study the stable dimension of $A$, which is the dimension of the stable module category of $A$ in the sense of Rouquier. Then we prove that $A$ is representationfinite if the stable dimension of $A$ is zero.
\end{abstract}

\section{$\S 1$. Introduction}

In [10], Rouquier introduced the notion of dimension of a triangulated category. One of his aims was to give a lower bound for Auslander's representation dimension of self-injective algebras; namely, he showed that, for any non-semisimple self-injective finite-dimensional algebra $A$ over a field $k$, the representation dimension of $A$ is greater than or equal to the stable dimension of $A$ plus 2 (see [9]).

On the other hand, Auslander in [2] introduced the representation dimension, which he hoped might serve as a good measure of how far a representation-infinite algebra is from being representation-finite. Actually, he showed that for any Artin algebra $\Lambda, \Lambda$ is representation-finite if and only if it has representation dimension at most 2 .

Thus, any non-semisimple representation-finite self-injective finite-dimensional algebra over a field $k$ must have stable dimension zero, which also follows immediately from the definition. Then a natural question arises as to whether the converse should also hold.

The aim of this article is to prove that the converse holds provided that $k$ is algebraically closed. Namely, our main theorem (see Theorem 3.9) is the following.

Main Theorem. Let $A$ be a non-semisimple self-injective finite-dimensional connected algebra over an algebraically closed field $k$. If the set $_{s} \Gamma(A)_{0}$

Received April 5, 2010. Revised November 20, 2010. Accepted December 22, 2010.

2010 Mathematics Subject Classification. Primary 16G60; Secondary 18 E30.

(C) 2011 by The Editorial Board of the Nagoya Mathematical Journal 
of isoclasses of nonprojective indecomposable A-modules admits only finitely many $\Omega$-orbits, then $A$ is representation-finite.

As a corollary, we have the desired result (see Corollary 3.10).

Main Corollary. If the stable dimension of $A$ is zero, then $A$ is representation-finite.

Although the consensus among some experts was that this statement should be true, proof of that has not been reached before this paper.

\section{$\S 2$. Stable dimension}

Let $\mathcal{T}$ be a triangulated category with shift functor $\mathrm{T}$. And let $\mathcal{I}$ and $\mathcal{J}$ be full subcategories of $\mathcal{T}$. We denote by $\langle\mathcal{I}\rangle$ the smallest full subcategory of $\mathcal{T}$ containing $\mathcal{I}$ and which is closed under shifts, finite direct sums, direct summands, and isomorphisms. Moreover, we denote by $\mathcal{I} * \mathcal{J}$ the full subcategory of $\mathcal{T}$ consisting of objects $M \in \mathcal{T}$ for which there exists a triangle $I \rightarrow M \rightarrow J \rightarrow \mathrm{T} I$ with $I \in \mathcal{I}$ and $J \in \mathcal{J}$. Put $\mathcal{I} \diamond \mathcal{J}=\langle\mathcal{I} * \mathcal{J}\rangle$, and inductively we define

$$
\langle\mathcal{I}\rangle_{n+1}= \begin{cases}\langle\mathcal{I}\rangle & \text { if } n=0, \\ \langle\mathcal{I}\rangle_{n} \diamond\langle\mathcal{I}\rangle & \text { if } n \geq 1 .\end{cases}
$$

Then the dimension of $\mathcal{T}$ is

$$
\operatorname{dim} \mathcal{T}:=\min \left\{n \geq 0 \mid\langle M\rangle_{n+1}=\mathcal{T} \text { for some } M\right\}
$$

or $\infty$ when there is no such object $M$ for any $n$.

Let $A$ be a non-semisimple self-injective finite-dimensional algebra over a field $k$. We denote by $A$-mod the abelian category of finite-dimensional (over $k$ ) left $A$-modules, and we denote by $A$-mod the stable module category of $A$, whose objects are the same as those of $A$-mod and morphisms from $X$ to $Y$ are given by the quotient space $\operatorname{Hom}_{A}(X, Y) / \mathrm{P}(X, Y)$, where $\mathrm{P}(X, Y)$ consists of those morphisms from $X$ to $Y$ which factor through a projective $A$-module. The syzygy functor $\Omega: A$ - $\underline{\bmod } \rightarrow A$ - $\underline{\bmod }$ is a functor defined by the correspondence of an object $X$ to the kernel of the projective cover of $X$. Note that if $X$ is indecomposable, then $\Omega(X)$ is also indecomposable. Moreover, note that the syzygy functor $\Omega$ is an equivalence, and the stable module category $A$-mod is a triangulated category with shift functor $\Omega^{-1}$ (see [5]). Then we define the stable dimension of self-injective algebras. 
Definition 2.1. The stable dimension of $A$ is

$$
\text { stab. } \operatorname{dim} A:=\operatorname{dim}(A-\underline{\bmod }) \text {. }
$$

Remark 2.2. Let $A$ and $B$ be non-semisimple self-injective finite-dimensional algebras over a field $k$. If $A$ and $B$ are stably equivalent as triangulated categories (that is, $A$-mod and $B$ - $\underline{\bmod }$ are equivalent as triangulated categories), then stab. $\operatorname{dim} A$ and stab. $\operatorname{dim} B$ coincide. For example, if $A$ and $B$ are derived equivalent, then they are stably equivalent as triangulated categories (see [8]), and hence stab. $\operatorname{dim} A$ and $\operatorname{stab} . \operatorname{dim} B$ coincide. Note that if $k$ is an algebraically closed field and if either $A$ or $B$ is representationfinite, they are derived equivalent if and only if they are stably equivalent as triangulated categories (see [1]).

The representation dimension of a non-semisimple Artin algebra $\Lambda$ is rep $\cdot \operatorname{dim} \Lambda:=\min \left\{\mathrm{gl} . \operatorname{dim} \operatorname{End}_{\Lambda}(M) \mid M:\right.$ a generator and a cogenerator $\}$.

(For a semisimple Artin algebra, this value is defined to be 1.)

Rouquier has shown the following result.

Proposition 2.3 ([10, Proposition 3.9], see also [2, second proposition, p. 559]). Let $A$ be a non-semisimple self-injective finite-dimensional algebra over a field. Then

$$
\mathrm{LL}(A) \geq \operatorname{rep} \cdot \operatorname{dim} A \geq \operatorname{stab} \cdot \operatorname{dim} A+2
$$

where the Loewy length $\operatorname{LL}(A)$ is the smallest integer $r$ such that $\operatorname{rad}(A)^{r}=0$.

After Auslander proved in [2, second proposition, p. 559] that $\operatorname{LL}(A)+1 \geq$ $\operatorname{rep} \cdot \operatorname{dim} A$, Rouquier has improved it by indicating that the equality does not occur, and hence the first inequality in Proposition 2.3 holds.

REMARK 2.4. The stable dimension is always finite by the first inequality in Proposition 2.3. Recall also that for any Artin algebra, the representation dimension is always finite (see [6]).

In [2], Auslander has also shown the following result.

Theorem 2.5 ([2, first proposition, p. 559]). Let $\Lambda$ be an Artin algebra. Then $\Lambda$ is representation-finite if and only if $\operatorname{rep} . \operatorname{dim} \Lambda \leq 2$.

Thus we observe that any (non-semisimple) representation-finite selfinjective finite-dimensional algebra over a field $k$ has stable dimension zero. The converse of this has been held to be true, but this is nontrivial. We therefore present an argument to affirm that this is the case when $k$ is an algebraically closed field. 


\section{§3. Main results}

Throughout the remainder of this article, $k$ denotes a fixed algebraically closed field, and all algebras are finite-dimensional associative $k$-algebras with an identity.

For any $k$-algebra $A$, we denote by $\Gamma(A)$ the Auslander-Reiten quiver of $A$ whose vertices are isoclasses of indecomposable (left) $A$-modules and arrows are irreducible maps. We may identify the vertices of $\Gamma(A)$ with the indecomposable $A$-modules. Then we have the Auslander-Reiten translation $\tau=D \operatorname{Tr}$ and $\tau^{-1}=\operatorname{Tr} D$, where $D: A-\bmod \rightarrow A^{\mathrm{op}}$-mod is the standard duality $\operatorname{Hom}_{k}(-, k)$.

In the rest of our paper, we assume that $A$ is a non-semisimple selfinjective connected algebra, unless otherwise stated. Thus we have $\tau \cong$ $\Omega^{2} \nu \cong \nu \Omega^{2}$ since $\Omega \nu \cong \nu \Omega$, where $\nu=D \operatorname{Hom}_{A}(-, A)$ is the Nakayama functor.

In addition, we denote by ${ }_{s} \Gamma(A)$ the stable Auslander-Reiten quiver of $A$, obtained from $\Gamma(A)$ by removing the projective-injective vertices and the arrows attached to them. Then the set ${ }_{s} \Gamma(A)_{0}$ of vertices of ${ }_{s} \Gamma(A)$ coincides with the set of isoclasses of nonprojective indecomposable $A$-modules. It is well known that we can recover $\Gamma(A)$ from ${ }_{s} \Gamma(A)$. For a connected component $C$ of $\Gamma(A)$, we denote by ${ }_{s} C$ the stable part of $C$. Note that the Auslander-Reiten translation $\tau$ is an automorphism of the quivers ${ }_{s} \Gamma(A)$ and ${ }_{s} C$, and its inverse $\tau^{-1}$.

Then we mention the following fundamental fact on self-injective algebras of stable dimension zero.

LEMMA 3.1. The following are equivalent:

1. stab. $\operatorname{dim} A=0$,

2. ${ }_{s} \Gamma(A)_{0}$ admits only finitely many $\Omega$-orbits.

Proof. By definition, the assertion follows from the fact that stab . $\operatorname{dim} A=$ 0 implies $A$ - $\underline{\bmod }=\langle M\rangle=\operatorname{add}\left\{\Omega^{i} M \mid i \in \mathbb{Z}\right\}$ for some $M \in A$ - $\underline{\bmod }$.

An indecomposable $A$-module $M$ is called $\tau$-periodic if $\tau^{n} M \cong M$ for some $n>0$. Then it is easy to prove the following lemma on a connected component of $\Gamma(A)$ containing a $\tau$-periodic module.

Lemma 3.2. Suppose that a connected component $C$ of $\Gamma(A)$ contains a $\tau$-periodic module. Then all modules in ${ }_{s} C$ are $\tau$-periodic. 
Given an indecomposable $A$-module $M$, we denote by $\tau^{\mathbb{Z}} M$ the $\tau$-orbit of $M$, that is, $\tau^{\mathbb{Z}} M:=\left\{\tau^{n} M \mid n \in \mathbb{Z}\right\}$. Similarly, the $\Omega$-orbit of $M$ and the $\langle\tau, \Omega\rangle$-orbit of $M$ are denoted by $\Omega^{\mathbb{Z}} M:=\left\{\Omega^{m} M \mid m \in \mathbb{Z}\right\}$ and $\tau^{\mathbb{Z}} \Omega^{\mathbb{Z}} M:=$ $\left\{\tau^{n} \Omega^{m} M \mid n, m \in \mathbb{Z}\right\}$, respectively.

For a set $S$ of indecomposable $A$-modules, we denote by $\mathrm{d}(S)$ the supremum of $k$-dimensions of modules in $S$, namely,

$$
\mathrm{d}(S):=\sup _{X \in S}\left(\operatorname{dim}_{k} X\right)
$$

Then we have the following.

Lemma 3.3. If an indecomposable A-module $M$ is $\tau$-periodic, then $\mathrm{d}\left(\Omega^{\mathbb{Z}} M\right)<\infty$.

Proof. By the hypothesis, there exists $n>0$ such that $\tau^{n} M \cong M$. Since $\tau \cong \Omega^{2} \nu$, we have $\nu^{n} M \cong \Omega^{-2 n} M$. Since the Nakayama functor $\nu$ preserves the dimension of modules, $\operatorname{dim}_{k} \Omega^{i} M$ is determined by $i$ modulo $2 n$. The assertion follows.

Therefore, we have the following fact.

LEMmA 3.4. If ${ }_{s} \Gamma(A)_{0}$ admits only finitely many $\Omega$-orbits, then

$$
\mathrm{d}\left(\left\{\text { all } \tau \text {-periodic modules in }{ }_{s} \Gamma(A)\right\}\right)<\infty \text {. }
$$

Proof. Let $M_{1}, \ldots, M_{l}$ be a complete list of representatives of $\Omega$-orbits of $\tau$-periodic modules in ${ }_{s} \Gamma(A)_{0}$. Note that if $M$ is $\tau$-periodic, then all modules in $\Omega^{\mathbb{Z}} M$ are $\tau$-periodic. Then $\bigcup_{i} \Omega^{\mathbb{Z}} M_{i}$ is just the set of all $\tau$ periodic modules in ${ }_{s} \Gamma(A)$. By Lemma 3.3, we have

$$
\begin{aligned}
\mathrm{d}\left(\left\{\text { all } \tau \text {-periodic modules in }{ }_{s} \Gamma(A)\right\}\right) & =\mathrm{d}\left(\bigcup_{i} \Omega^{\mathbb{Z}} M_{i}\right) \\
& =\sup _{i} \mathrm{~d}\left(\Omega^{\mathbb{Z}} M_{i}\right)<\infty,
\end{aligned}
$$

as desired.

Now, we mention the following well-known fact on representation-finite algebras, due to Auslander.

Lemma 3.5 (Auslander [3, Chapter VI.1, Theorem 1.4]). Let A be a finitedimensional connected $k$-algebra, and let $C$ be a connected component of $\Gamma(A)$. If $\mathrm{d}(C)<\infty$, then $A$ is representation-finite. 
By using this lemma, we prove the following key proposition.

Proposition 3.6. Assume that ${ }_{s} \Gamma(A)_{0}$ admits only finitely many $\Omega$ orbits. If there exists a $\tau$-periodic module in ${ }_{s} \Gamma(A)$, then $A$ is representationfinite.

Proof. Let $X$ be a $\tau$-periodic module in ${ }_{s} \Gamma(A)$, and let $C$ be a connected component of $\Gamma(A)$ containing $X$. By Lemma 3.2, all modules in ${ }_{s} C$ are $\tau$-periodic. Note that there exist only finitely many projective-injective vertices. Then by Lemma 3.4, we have $\mathrm{d}(C)<\infty$, and thus $A$ is representationfinite by Lemma 3.5.

Here, we mention the following fact on non- $\tau$-periodic modules.

Lemma 3.7. Let $M$ be an indecomposable A-module. Assume that there exists a positive integer $n$ such that $\nu^{n} M \in \Omega^{\mathbb{Z}} M$. If $M$ is not $\tau$-periodic, then $\tau^{\mathbb{Z}} \Omega^{\mathbb{Z}} M$ is a union of finite number of $\tau$-orbits.

Proof. Let $m$ be an integer such that $\nu^{n} M \cong \Omega^{m} M$. By the hypothesis we have

$$
\tau^{n} M \cong \Omega^{2 n} \nu^{n} M \cong \Omega^{2 n+m} M(\nsubseteq M)
$$

with $2 n+m \neq 0$. Put $t:=|2 n+m|$. Then

$$
\Omega^{\mathbb{Z}} M \subset \tau^{n \mathbb{Z}} M \cup \tau^{n \mathbb{Z}} \Omega M \cup \cdots \cup \tau^{n \mathbb{Z}} \Omega^{t-1} M,
$$

and thus we have

$$
\tau^{\mathbb{Z}} \Omega^{\mathbb{Z}} M=\tau^{\mathbb{Z}} M \cup \tau^{\mathbb{Z}} \Omega M \cup \cdots \cup \tau^{\mathbb{Z}} \Omega^{t-1} M,
$$

as desired.

Moreover, we need Liu's characterization of representation-finite algebras over an algebraically closed field to prove our main theorem.

Lemma 3.8 ([7, proposition, p. 52]). Let A be a finite-dimensional algebra over an algebraically closed field $k$. Then $A$ is representation-finite if and only if $\Gamma(A)$ admits only finitely many $\tau$-orbits.

This follows from the second Brauer-Thrall conjecture. Therefore, the assumption that the base field $k$ is algebraically closed is essential.

Now we prove our main theorem. 
THEOREM 3.9. Let A be a non-semisimple self-injective finite-dimensional connected algebra over an algebraically closed field $k$. If the set ${ }_{s} \Gamma(A)_{0}$ of isoclasses of nonprojective indecomposable A-modules admits only finitely many $\Omega$-orbits, then $A$ is representation-finite.

Proof. Let $M_{1}, \ldots, M_{l}$ be a complete list of representatives of $\Omega$-orbits in ${ }_{s} \Gamma(A)_{0}$. Note that for each $i \in\{1, \ldots, l\}$ there exists a positive integer $n_{i}$ such that $\nu^{n_{i}} M_{i} \in \Omega^{\mathbb{Z}} M_{i}$.

If there exists an integer $s \in\{1, \ldots, l\}$ such that $M_{s}$ is $\tau$-periodic, then by Proposition 3.6, $A$ is representation-finite.

Then we can assume that each $M_{i}$ is not $\tau$-periodic. By Lemma 3.7, $\Gamma(A)$ admits only finitely many $\tau$-orbits. Thus $A$ is representation-finite by Lemma 3.8, as desired.

By Lemma 3.1, we obtain the following result.

CoRollary 3.10. If the stable dimension of $A$ is zero, then $A$ is representation-finite.

Proof. Suppose that stab. $\operatorname{dim} A=0$. Then by Lemma 3.1, ${ }_{s} \Gamma(A)_{0}$ admits only finitely many $\Omega$-orbits, and thus $A$ is representation-finite by Theorem 3.9 .

Furthermore, we obtain the following result.

Corollary 3.11. If rep. $\operatorname{dim} A=3$, then $\operatorname{stab} . \operatorname{dim} A=1$.

Proof. If rep $\operatorname{dim} A=3$, then stab. $\operatorname{dim} A \leq 1$ and $A$ is not representationfinite by Proposition 2.3 and Theorem 2.5. Then we have, by Corollary 3.10, stab. $\operatorname{dim} A=1$.

It has been conjectured whether the following holds.

ConjeCtuRe 1. Any algebra of tame representation type has representation dimension at most 3 .

Moreover, we have a new conjecture by Corollary 3.11.

ConjeCture 2. Any (non-semisimple) self-injective k-algebra of tame representation type has stable dimension at most 1 .

Conjecture 1 is true for some classes of tame algebras, such as domestic self-injective algebras socle equivalent to a weakly symmetric algebra of Euclidean type (see [4]). Thus, by Proposition 2.3, these algebras have stable dimension at most 1 . 
Note that in general the converse of Conjecture 1 does not hold. Any wild hereditary algebra is a counterexample.

Similar to Conjecture 1, the converse of Conjecture 2 does not hold in general; for instance, any wild self-injective finite-dimensional algebra over a field with radical cube zero has stable dimension at most 1 (see Proposition 2.3).

Acknowledgments. The author would like to thank Takuma Aihara for first suggesting this problem. He also expresses his sincere gratitude to Osamu Iyama for helpful discussions and comments and to Hideto Asashiba for helpful advice.

\section{REFERENCES}

[1] H. Asashiba, The derived equivalence classification of representation-finite selfinjective algebras, J. Algebra 214 (1999), 182-221.

[2] M. Auslander, "Representation dimension of Artin algebras" in Selected Works of Maurice Auslander, Pt.1, Amer. Math. Soc., Providence, 1999.

[3] M. Auslander, I. Reiten and S. O. Smalø, Representation Theory of Artin Algebras, Cambridge Stud. Adv. Math. 36, Cambridge University Press, Cambridge, 1995.

[4] R. Bocian, T. Holm, and A. Skowroński, The representation dimension of domestic weakly symmetric algebras, Cent. Eur. J. Math. 2 (2004), 67-75.

[5] D. Happel, Triangulated Categories in the Representation Theory of Finitedimensional Algebras, London Math. Soc. Lecture Note Ser. 119, Cambridge University Press, Cambridge, 1988.

[6] O. Iyama, Finiteness of representation dimension, Proc. Amer. Math. Soc. 131 (2003), 1011-1014.

[7] S. Liu, Degrees of irreducible maps and the shapes of Auslander-Reiten quivers, J. London Math. Soc. (2) 45 (1992), 32-54.

[8] J. Rickard, Derived categories and stable equivalence, J. Pure Appl. Algebra 61 (1989), 303-317.

[9] R. Rouquier, Representation dimension of exterior algebras, Invent. Math. 165 (2006), 357-367.

[10] , Dimensions of triangulated categories, J. K-Theory 1 (2008), 193-256; Correction, J. K-Theory $\mathbf{1}$ (2008), 257-258.

Department of Mathematics and Physics

Graduate School of Science

Osaka City University

Sumiyoshi-ku, Osaka 558-8585

Japan

yosiwaki@sci.osaka-cu.ac.jp 